\title{
Molecular Profiling for CUP Cancers: Are We There Yet?
}

\author{
Gauri Varadhachary \\ Department of Gastrointestional Medical Oncology, M.D. Anderson Cancer Center, University of Texas, Houston, TX, USA
}

In this issue of Onkologie, Gross-Goupil and colleagues, through their prospective study and case discussions draw our attention to an important emerging field: molecular profiling of carcinoma of unknown primary (CUP) [1]. The authors describe a feasibilty study using the CupPrint ${ }^{\circledR}$ assay (Agendia BV, Amsterdam, The Netherlands) in 22 patients with confirmed CUP cancers. CupPrint is an oligonucleotide microarray that contains 495 genes that were selected as highly differentially expressed between 48 tumor types. In 2008, Horlings and colleagues initially reported the results for this assay in 84 patients with known primary adenocarcinomas and in 38 CUP patients [2]. The gene expression based assay classified the primary site correctly in $83 \%$ of the known cancers. Of the 38 patients with CUP, extensive immunohistochemistry (IHC) classified 16 patients and CupPrint identified most (94\%) of these. In the remaining CUP patients, the cancer remained unclassified and CupPrint was beneficial in 14 of these 22 CUP patients (64\%). The study described herein is a prospective feasibility study in 22 pts with CUP using the CupPrint assay.

Utilizing molecular profiling in CUP is based on the premise that accurate diagnosis of the primary site of origin in CUP would impact patients' prognosis and management, allowing site specific therapy. This becomes especially important with the continued emergence of effective therapies targeted against specific tumor types. Several genomic technologies which provide large scale gene expression profiles based on mRNA or microRNA are currently in commercial application. The feasibility of utilizing mRNA / microRNA from small biopsy samples available as formalin-fixed and paraffin embedded (FFPE) tissue (using a quantitative reverse transcriptase-polymerase chain reaction [qRT-PCR] platform), and the reasonable turn-around time (11 days in this study) makes it practical for clinical use. One challenge is that it is not unusual to have insufficient tumor quantity (block exhaustion) or poor RNA preservation in about $15-20 \%$ of samples in clinical practice. In the current study, analysis was not feasible in 4 patients $(18 \%)$.
At present, light microscopy with hematoxylin and eosin staining and IHC form the backbone of CUP pathologic work up. IHC in CUP cancers perform the best when used in groups which gives rise to patterns that are strongly indicative of certain profiles and can assist with treatment planning (e.g. TTF-1/CK7+, Ck20+/CDX-2+/CK7- phenotypes have been reported as very suggestive of lung and lower gastrointestinal cancer profiles respectively). Several groups have approached IHC with decision trees based on their specificity, sensitivity, and predictive values (moving from most specific to the least or from the highest positive predictive value to lowest) [3]. Even with sophisticated decision trees, IHC is not without its limitations - several factors affect tissue antigenicity (retrieval, processing, and fixation), interpretation of IHC in tumor vs. normal tissue, inter- and intraobserver variability and tissue heterogeneity and inadequacy (given small biopsy sizes). Communication between the clinician and pathologist is essential and cannot be replaced by an exhaustive battery of stains. In the era of sophisticated IHC, we need to judiciously select patients who will benefit the most from molecular profiling. In the study reported by Gross-Goupil and colleagues, lung and colorectal cancer profiles were the most commonly identified tissues of origin (as noted with other studies) although IHC data on these reported cases is absent to assess concordance with pathology.

Although useful in some subtypes, the current utility of the profiling approach is probably limited by the paucity of effective drugs for several CUP cancer profiles. As novel therapies are developed for additional known cancers, profiling may significantly impact the appropriate CUP subtypes. Additionally, profiling assay results may also direct the use of specific molecular tests that may influence first or subsequent lines of therapy (e.g. Her-2, IHC/FISH, EGFR or ALK mutational analysis). Currently, profiling assays have their niche with patients who have a broad IHC differential or no helpful IHC data. This is especially a challenge when two completely different therapies are indicated based on pathology [4]. The authors have planned a study that evaluates the clinical utility

\section{KARGER \\ Fax +497614520714 \\ Information@Karger.de}

www.karger.com
(C) 2012 S. Karger GmbH, Freiburg

0378-584X/12/0351-0011\$38.00/0

Accessible online at:

www.karger.com/onk
Gauri Varadhachary, M.D.

University of Texas, M.D. Anderson Cancer Center

Dept. of Gastrointestional Medical Oncology

1515 Holcombe Blvd. Unit 426; Houston, TX 77030, USA

Tel. +1 713 792-2828, Fax -0539

gvaradha@mdanderson.org 
of profiling in a randomized trial comparing progression free survival outcomes in patients receiving therapy based on site of origin prediction versus those receiving standard empiric therapies (GEFCAPI-04). This is an important study and probably warranted, although it is a challenging task due to the very heterogeneous presentations of CUP cancers. Going forward, we can envision using an integrated cost and clinically effective algorithm in selected patients - i.e. one that identifies a patient's CUP profile using IHC and profiling efficiently and where it matters the most.

1 Gross-Goupil M, Massard C, Lesimple T, et al.: Identifying the primary site using gene expression profiling in patients with carcinoma of an unknown primary (CUP): a feasibility study from the GEFCAPI. Onkologie 2011;35: DOI: $10.1159 / 000336300$.

2 Horlings HM, van Laar RK, Kerst JM, et al.: Gene expression profiling to identify the histogenetic origin of metastatic adenocarcinomas of unknown primary. J Clin Oncol 2008;26:4435-41.

3 Dennis JL, Hvidsten TR, Wit EC, et al.: Markers of adenocarcinoma characteristic of the site of origin: development of a diagnostic algorithm. Clin Cancer Res 2005;11:3766-72.

4 Varadhachary GR, Spector Y, Abbruzzese JL, et al.: Prospective gene signature study using microRNA to identify the tissue of origin in patients with carcinoma of unknown primary. Clin Cancer Res 2011;15;17:4063-70. 